\title{
How to Set Up a Community Health Venture: Lessons from 10 Years in Kenya
}

\author{
Irena Gorski \\ Pennsylvania State University \\ University Park, PA
}

\author{
Stephen Suffian \\ Pennsylvania State University \\ University Park, PA
}

\author{
Joshua T. Bram \\ Pennsylvania State University \\ University Park, PA
}

\author{
Phoebe Canagarajah \\ Pennsylvania State University \\ University Park, PA
}

\author{
Khanjan Mehta \\ Pennsylvania State University \\ University Park, PA \\ khanjan@engr.psu.edu
}

Abstract - Despite high potential, many community health ventures in developing countries fail to evolve beyond the pilot stage. A fundamental problem is that many ventures utilize community health workers (CHWs) as volunteers, but they leave to generate a living income for their families. After ten years of experience running a community health venture in East Africa, while observing other ventures come and go, several factors have emerged as essential for any venture to achieve success. This article presents a methodology to set up a community health venture where CHWs generate income from their work while improving health outcomes for members of their communities. The authors seek to provide individuals working to solve last-mile healthcare challenges with a practical guide for establishing community health systems and navigating the validation, networking, planning, launch, and execution phases. The goal for the ventures developed using this methodology is to operate in an economically sustainable way.

Index Terms - community health workers, self-sustainable, telemedicine, venture

\section{INTRODUCTION}

Developing countries face dual obstacles to improving human health: a disproportionate burden of disease and a shortage of healthcare workers. Community health ventures with Community Health Workers (CHWs) have emerged as a promising solution to provide primary care at the community level in developing countries. ${ }^{1,2}$ A community health venture is an undertaking by an organization established by a social entrepreneur that provides healthcare services for people in a specific region. For example, Djantoli is a community health venture that operates in Mali; its CHWs visit children at home, collect simple health data, and provide some education on illness prevention and nutrition. ${ }^{3}$ The information is collected on the CHWs' phones and delivered remotely to a doctor who identifies children at risk and encourages them to see a local doctor, which is covered by a subscription to Djantoli's services.

CHWs can provide services to communities previously disconnected from the healthcare system, including illness prevention, basic treatment, hygiene and sanitation, data collection, and health education. Establishing community health ventures provides a mechanism and pathway for data collection, aggregation, and digitization by employing CHWs to use digital tools such as thermometers, heart rate monitors, cell phones, and tablets. Despite high potential, many 
International Journal for Service Learning in Engineering,

Humanitarian Engineering and Social Entrepreneurship

Vol. 11, No. 2, pp. 72-86, Fall 2016

ISSN 1555-9033

community health ventures fail following initial implementation. Pilot projects are often shortlived because they struggle to sustain themselves once initial seed funding has ended. ${ }^{4,5} \mathrm{~A}$ fundamental problem is that CHWs are volunteers and hence attrition rates are high because they need to generate a living income for their families.

In an effort to maximize the potential of a community health venture, the Humanitarian Engineering and Social Entrepreneurship (HESE) program at the Pennsylvania State University established a venture in Kenya called Mashavu. After several fast failures and iterations, Mashavu now employs six CHWs with a cash-positive revenue model. ${ }^{6}$ Through ten years of working with Mashavu while observing other community health ventures come and go, many factors have emerged as essential for the successful implementation of a community health venture. Success factors include support from the government; acceptance from the public; availability of technological infrastructure, including hardware, software and adequate bandwidth; sustainable funds and funding models; and appropriately trained health professionals or community health workers. ${ }^{7}$

How do we enable CHWs to engage in income-generating activities alongside their work? In response to over one hundred emails received by the Mashavu team asking how to set up, this article synthesizes lessons learned over ten years of piloting and implementation. This article presents a methodology to set up a community health venture where CHWs generate income from their work with collecting, aggregating, and digitizing health data while improving health outcomes for members of their communities. The authors seek to provide individuals working to solve last-mile healthcare challenges with a practical guide for establishing community health systems and navigating the planning, initial testing, and operation phases. The goal for the ventures developed using this methodology is to operate in an economically sustainable way.

This methodology is not a fool-proof formula for setting up a community health venture. It does, however, strive to illustrate the larger process of such a venture setup. In reality, there may be other aspects of the venture setup that are not mentioned in this article but may be needed to bring about sustainable health outcomes and/or have their CHWs generate income. This article provides a starting point for a conversation that needs to be undertaken by the right partners: CHWs, doctors, patients, local ministries of health, local NGOs, venture champions, and more. After providing a short background on Mashavu, this article explains the five phases of starting a venture from validation through execution, providing practical advice throughout each phase on avoiding common pitfalls and adopting best practices for success.

\section{MASHAVU 101}

Mashavu's initial pilot used computer-based kiosk systems to connect local community health workers to medical professionals. The system sent health information about height, weight, and blood pressure to medical professionals who then replied with recommendations to the CHWs. Mashavu served to improve access to healthcare, increase community health education, and socio-economic development in Kenya. ${ }^{8}$ During 2011, a pilot was conducted in five communities throughout Nyeri County, Kenya. Over the span of five months, Mashavu's CHWs met with 777 clients who paid for preventive health services. The venture's purpose was to act as a form of triage for clients in rural areas, offering recommendations for doctor visits based on a health survey and basic health data including blood pressure and temperature. One of the major outcomes of this venture was that $31 \%$ of clients were recommended for a doctor's visit for 
possible hypertension, a preventable non-communicable disease that has rising occurrence in Kenya. ${ }^{6}$

At the completion of the pilot, it was determined that this iteration of Mashavu, relying on a full-time paid nurse and stationary kiosks, was unsustainable. Rural areas were too sparse to keep CHWs busy. Further, it was found that few clients requested nurse feedback and paying the nurse was expensive. They overwhelmingly preferred quick blood pressure and BMI measurements rather than filling out long medical surveys and waiting for a response. A new iteration of Mashavu was then implemented where CHWs carried smartphones and a backpack containing an automatic blood pressure cuff, a weighing scale, a tape measure, and relevant supplies. The CHWs were also provided additional training and access to a custom electronic medical records platform. Rather than stay in stationary kiosks in rural areas, the technology shift and backpack enabled health workers to walk around the more populated Nyeri town. During this iteration it was realized that the data collected was not being utilized in the field. Clients were hesitant to have their data put on the cloud, and the use of technology continued to prevent an economically sustainable model. The next and final iteration proved to be sustainable. By replacing smartphones with carbon copy receipt books and switching to a new business model, the venture was able to provide health data to clients with CHW autonomy. These changes enabled the CHWs to see a high volume of clients, which in turn resulted in daily profits, eliminating the need for daily subsidies. In the final and current business model, the CHWs pay a portion of their income to Mashavu to work towards ownership of the medical devices. Currently, the venture employs six Mashavu Health Workers that provide 'know your numbers' service - providing blood pressure, temperature, waist circumference, and weight information to people in the Nyeri area and outlying villages, with a cash-positive revenue model. ${ }^{6} \mathrm{CHWs}$ provide input on what these numbers could mean and indicate to the clients when they should consider going to the doctor, which is of great value since they would otherwise not likely consider going. The venture was validated by the Kenyan Ministry of Public Health and Sanitation (MOPHS). They found that Mashavu's services in rural areas effectively extended the reach of MOPHS well beyond their infrastructure's capacity by generating extensive information on the residents' health history. This allowed the MOPHS to make a community diagnosis and undertake targeted preventive health campaigns. In addition, Mashavu successfully created the conditions for their CHWs to generate income.

\section{Launch Process of InCome-Generating Community Health System}

The first phases in establishing a community health system include: a validation phase; a networking and planning phase; a consideration of whether to follow a professional health worker-based track and/or community health worker (CHW) track; a venture launch preparation phase; and the execution of the community health venture (Figure 1). 


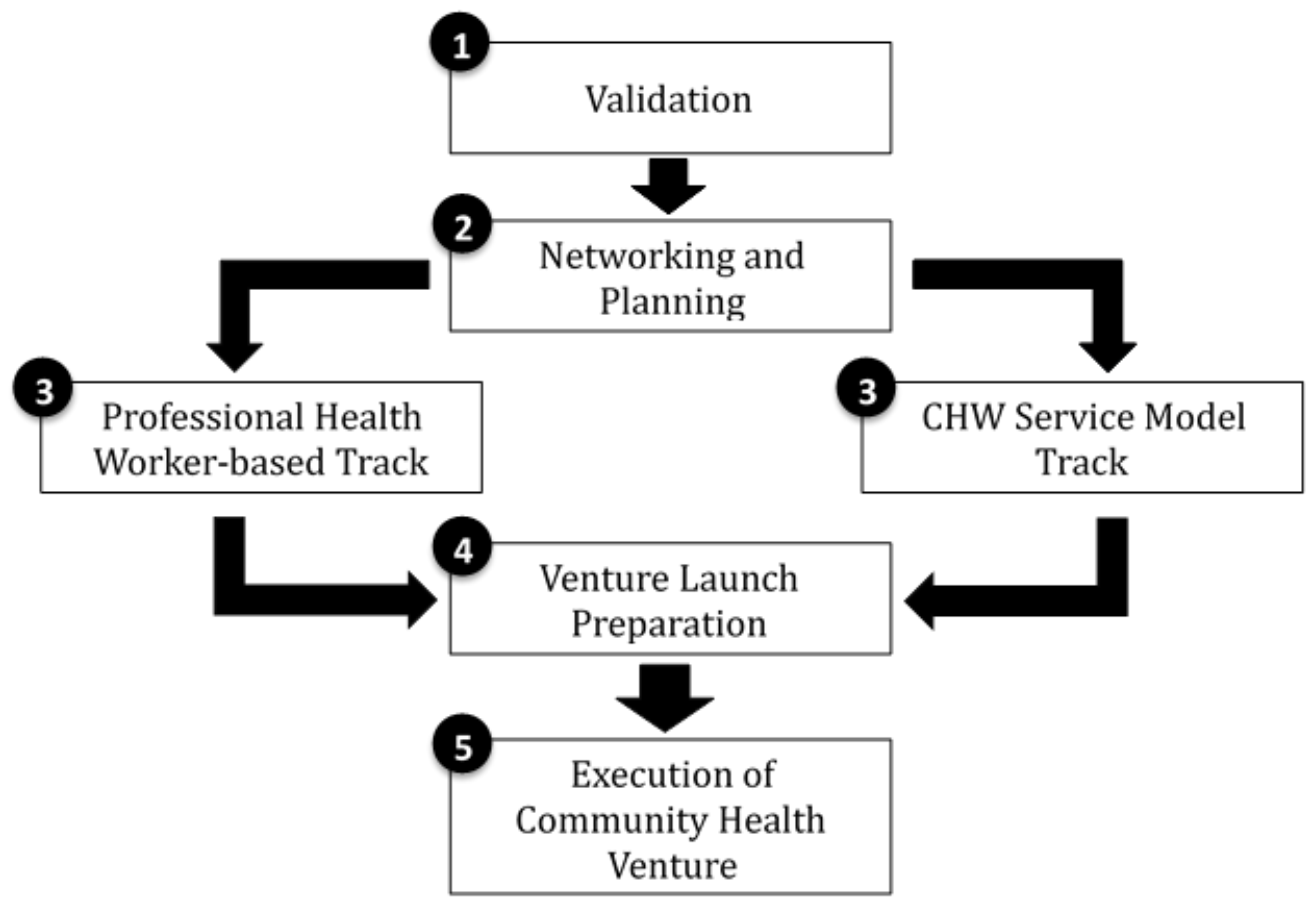

FIGURE 1

Five Main Phases of Community Health Venture SetuP.

\section{Part 1 Of 5: Gain Validation}

When a health venture is first hypothesized as a potential solution to an unmet need, the following steps explain how to determine if there is in fact such a need for a venture.

1. Conceptualize Project and Necessity of System. Upon conceptualizing of a project, an analysis of the system it fits into is warranted. The following steps describe the process of such an analysis.

- Conduct landscape analysis. Research other ventures, particularly those focused on telemedicine and community healthcare that are already located in the region so as to avoid areas where solutions exist, preventing a potential waste of time and effort.

- Study the local CHW system. Learn how it is set up, what their current responsibilities are, and if they already have any income-generating activities.

- Review literature. Preliminarily identify ailments affecting the specific areas, particularly for identifying underlying socioeconomic issues. These records are typically at the local public health office.

- Gain an understanding of the current healthcare system. Determine the specific services offered by hospitals and other healthcare organizations that can be leveraged for the venture and which gaps can be addressed through the venture. 
2. Validate the Potential for a System and Consider System Design Criteria. Once an additional healthcare system is identified as a necessity and it is clear there is no system in place that can currently satisfy the demand for improved healthcare or affect the proper changes necessary, several questions should be addressed:

- What healthcare gap will the potential venture fill?

- Is there a community interest in this venture?

- Are there teachable individuals who can learn about public health and become champions for the venture?

- What contextually specific problems will inform the design of this system?

- What current programs or systems are in place that will make integration of the venture a smoother process?

- Which potential stakeholders in the region will be interested in working with the venture?

3. Engage Local Partners. Once potential stakeholders have been identified that will likely be interested in the program or will directly benefit from the venture, they should be approached about how they can be involved with the setup.

- Engage the government health ministry. The health challenges and goals of the ministry should be discussed so that the venture can align its mission with that of the government. On-going governmental health initiatives that can lend their support to the venture are naturally of interest, as is a list of communities in a country with little to no healthcare support because these make ideal targets for a community health venture.

- Reach out to other non-profits and aid organizations. Some of these groups have been in the region for an extended period of time and will understand best how to work through any bureaucratic or community issues that may arise relating to healthcare and social work.

- Obtain all necessary agreements. At this point in time, obtain necessary agreements with local stakeholders for collaborating and networking in the country. Permissions will need to be formalized later in the venture launch phase.

\section{PART 2 OF 5: NETWORK AND PLAN}

After validating the need and approach for the venture, you can begin to determine the network of people and resources that you need. These steps explain methods for considering what resources are necessary and finding relevant stakeholders who can help to best utilize those resources.

1. Determine technological capacities and strengths in the area. Temper the use of advanced technology with the abilities and preferences of the users. Due to the limited nature of cell phones in most communities in developing countries, consider:

- Store-and-forward mechanisms that send data to a central server as soon as the user reaches an area with adequate cell service. This will help the issue of lack of cell 
phone data storage as well as the high risk for losing data due to accidental clicking and data overwriting.

- Other telecommunication platforms besides text messaging, such as GPRS (General Packet Radio Service - cellular data) along with free messaging, email, and social media services. This will alleviate the problem of being forced to abbreviate health-related messages due to the restriction on the number of characters per text message.

- Easy-to-scan handwritten forms for optical scanning and data storage when the electronic health systems are not accessible.

- The venture's own modems and servers that have the capability to transmit, store, and analyse large amounts of client health information.

- Existing telecommunications platforms, social media, or older technologies can be a more effective and financially viable option than going with the most technologically advanced products or developing a new web service.

\section{Pursue economic sustainability for the venture through business model development and partnerships with existing businesses}

- Develop an appropriate cost structure. Several factors go into coming up with a proper end-user price. In some cases, it is not appropriate to charge end users a fee, but in many cases this is one of the few ways to guarantee long-term economic sustainability. A price should not create significant barriers, but should maintain appropriate incentives and generate income for the system operators. Variable pricing should be allowed so that operators can easily see people who can't afford the standard price. After a standard price is set, it is best to allow those running the system to take control of this flexibility. Factors to consider when deciding upon a price include the discretionary spending of people in the area, predicted demand for the system, and the appropriate income for a day's work.

- Find partners that will provide any services for lower costs. Free or discounted services can be justified to larger corporations as advertising through increased notoriety for its social awareness and concern for local communities. This should be completed once the venture is being launched and marketing is a priority.

- Form partnerships with local entrepreneurs and business owners. Such business locations provide for small but crucial opportunities, such as additional supply storage and cell phone charging. These partnerships can be lucrative between these established local businesses and the venture

- Locate external partners for funding. Community health systems need financial investment from outside sources, both at the onset of the venture and during the course of start-up operations. External capital is especially important in the early stages of operations when demand is low, the technology is still being tested, and few people are familiar with using the system. Often, the largest and most successful telemedicine ventures use a variety of government and corporate sponsors who are providing money in exchange for advertising rights, publicity, and public health interest. $^{10}$ 
- Decide on employees. The final venture business model will focus either on the use of professional healthcare staff or local community health workers to deliver the service. While professional staff may have more qualifications, they will require higher incomes and have busier schedules. CHWs have a limited amount of training, but are typically volunteers and are interested in income-generating activities that bolster their work.

3. Identify partner communities. The initial identification of partner communities to initiate the venture is incredibly important, as it will determine the sites of focus for all future iterations of the venture. This identification should be done through collaboration with public health officials who can provide a deeper contextual understanding of the people that need additional health services and support.

4. Talk with community members to facilitate integration of the venture into community life.

- Identify the proper professionals to contact for interviews by consulting with public health officials and local healthcare providers, such as doctors and nurses.

- Broadly talk with leaders about the venture including community leaders, business owners, and CHWs, detailing exactly what it has to offer a community and the vision plan for the future. At this point in time the venture should sell itself. It may be possible to engage the people further and catalyse the evolution of the venture by discussing and exploring potential community involvement opportunities.

- Gather all contact information for the proper administrators and meet in person with as many people as possible. These administrators include medical officers of health at the ministry, the Mayor or local council, village elders, and other community organization and business leaders. This contact information will be vital when problems arise during implementations that require authority figures to intervene.

- Hold focus groups to both evaluate the appropriateness of the venture and promote its efficacy. In this forum, all concerns about the venture can be discussed along with any context-specific concerns of the community about the venture's mission.

5. Based on the partners identified, develop a business plan for a pilot venture. After agreements are in place to get the venture up and running, a sustainable business plan should be established that considers all aspects of the venture's operation. The business plan should be developed in collaboration with the partner organization, whether that be the ministry of health, non-profit, or local businesses. During the creation of the business model, all aspects of a middle-to-low resource context should be considered, including:

- Limited customer financial resources

- Lack of start-up capital

- Local operating policies

- Community education

- Current health services

- Value created for the community

- Service delivery methods 
The most important piece of developing a business model is understanding that it should be a negotiation with the system operators, not an imposed business strategy. The model that works best is one that is well understood and executed by those running the system. It is important to be open to periodically adapting the business model in order to maintain the system and find the mode of operation that optimizes the chance for long-term sustainability.

\section{If solely employing CHWs, consider the following business models that have been successful before. ${ }^{10}$}

- Additional Services Business Model: The CHW is trained by the national CHW program but is provided with additional equipment and training by the venture. In addition to the CHW's usual routine services in the community, they offer additional services during their routine visits, which community members pay for. In order to quickly grow the customer base and scale the venture, CHWs can initially offer a service free-of-cost. Community health ventures must demonstrate the value of their services to the community, especially if there was an initial no-cost pilot in a particular community. The price point can be determined by pilot tests with paying customers rather than relying on surveys or assumptions.

- Additional Products Business Model: CHWs sell health products in addition to the basic services they already provide. Both product and price validation can come from surveys of current product prices, community interviews, and using local champions to determine where there are needs. While conducting routine services within the community, a CHW can sell additional products to community members for a small profit.

- Data Collection Business Model: While conducting routine services within the community, a CHW can collect pertinent information requested by the government, a for-profit, or a non-profit entity and be paid a stipend for their efforts. CHWs can use smartphones, tablets, or other digital health tools to collect, aggregate, and digitize data.

- Advertising Business Model: CHWs can advertise products and services of other companies in exchange for compensation. It is typically easier to find potential partners for advertising than those for data collection. With this model, an external entity trains CHWs and provides necessary advertising supplies. While the CHW is conducting routine services in the community, they can educate and advertise products to community members and receive a stipend from the external entity.

- Referral Business Model: CHWs make recommendations for individuals to engage with external organizations providing health services, i.e. a hospital, clinic, or other health care provider. The health care providers pay the CHWs to recommend community members to their facilities and the CHWs make recommendations through simple evaluations based on a community member's symptoms.

- Labour Business Model: CHWs can provide additional non-health-related services to community members that do not necessarily tie directly to health, including farming, cleaning, and laundry. These types of ventures provide help to those who may not be able to do these things themselves due to disease, age, or other health related concerns. With this model, the CHWs help community members with home 
activities and community members pay CHWs either through money or goods. External organizations may provide CHWs a stipend for activity completion.

- Educational Business Model: CHWs provide supplementary educational materials or services to community members on issues such as hygiene, diabetes, malaria, HIV, and many others. They are educated on relevant issues and often paid by the government's Ministry of Health, an NGO, community members or other external organizations.

- Regulatory Business Model: CHWs monitor the health of members of local organizations on behalf of NGOs or government entities. CHWs can also serve as health inspectors in charge of evaluating the hygiene of local businesses' employees. With this model, an organization trains CHWs to inspect and certify restaurants and other public establishments, enabling the CHWs to teach owners about proper hygiene and sanitation standards. CHWs are paid a stipend by the external entity managing the venture.

7. Create a more detailed venture model in concert with the local community leaders, taking into consideration the feedback you received in the focus groups.

- Begin to observe daily operations at community clinics. Observation can help in determining specific community needs to address through the venture.

- Discuss and agree upon with all partners the equity of work, finances, and time that each partner will contribute. Furthermore, a primary strategic goal, a definition of success, and a plan to scale should be discussed ahead of implementation to ensure that all partners are working with the same vision. This planning conversation can alleviate conflicting assumptions that may impede success later.

\section{PART 3 of 5: PILOT VENTURE}

At this point, a decision must be made regarding whether to use CHWs as mediators between patients and medical professionals or as the health professionals themselves. With a CHWmediated telemedicine track, CHWs will conduct the initial consultation and relay the health professional's reply. Choosing to follow this track will provide a greater degree of healthcare to the clients and will better inform clients as to their individual health, but will require greater resources.

If the venture lacks the funds or resources necessary to support a CHW-mediated telemedicine track, then having a track operated solely by CHWs will provide clients their basic health information and a convenient way to monitor their health. This is especially true for chronic issues such as diabetes, high blood pressure or high cholesterol. Hiring local citizens can help avoid language barriers and bridge tribal and ethnic differences. In practice, privacy expectations vary significantly from place to place, but some common trends like HIV status are almost never a part of patients' records and are not openly shared. CHWs already have privileged access to health information of people in their communities. It is best to conform with local norms and practices after consultation with the local health authorities. Both tracks will provide a revenue source to CHWs who are commonly volunteers. In order for either track to be successful, CHWs must be properly trained to provide community health services. The following will explain the common and distinct steps to take to pilot the venture based on Option 
A - Professional Health Worker, CHW-Mediated, Telemedicine Track - or Option B Independent CHW Service Model Track.

Steps 1 - 3 for both Option A (Professional Health Worker Track) and Option B (Independent CHW Service Model Track):

1. Recruit and train interested health professionals. Previously employed doctors, nurses, and/or CHWs should be retained and utilized for the training and fluid rollout of the community health venture.

- Explain the community health venture. This ensures that they understand what is expected of them in their new positions.

- Conduct interactive discussions on healthcare, community health, etc. This ensures that both the venture and the potential employees know what health problems need to be targeted.

- Administer a diagnostic test to CHWs. This assessment will ensure potential workers have a basic understanding of health and the venture. This diagnostic should include basic health knowledge, understanding of community health ventures, responsibility and community engagement.

- Select final doctors, nurses, and/or CHW employees. Employees who show selfmotivation, responsibility, and a good understanding of the venture's goals should be hired.

2. Identify a meeting space for training. This space can either be a classroom or a community's common gathering space. Ideally, it is easy to reach for most employees, well known, and centrally located.

\section{Customize a training curriculum}

- Provide training for all employees on venture operations and operating systems. This step should include training on the basic healthcare that they will carry out, any technology they will be utilizing, the data storage model, and how to relay diagnoses.

- Provide additional training for CHWs with visuals and hands-on application of learned concepts. The training curriculum could include the following:

- Health afflictions. This education would focus on the diseases CHWs will commonly encounter and assess. Education should take into account the knowledge CHWs already know from previous training and serve to complement that knowledge.

- Overcoming stigmas. For certain types of illnesses and diseases, there may be societal stigmas that community health ventures need to combat. Employees must be able to navigate difficult topics that require great amounts of trust with clients.

- Technology. In addition to the technology training provided to all employees, CHWs should be given hands-on training on how to use the technology they will use to gather health information.

- How to interact with clients. Training in this area should include modelling for CHWs how they are to act when they provide a client services. This includes but 
is not limited to: (1) how to greet a client and inform them of the services provided, (2) key questions to ask about a client's health history, (3) how to take a client's measurements and record health information, (4) how to assess whether a client needs to see a health professional, (5) how to explain to a client their health condition and if they need higher medical attention, and (6) how to charge for services.

\section{Steps 4-8 for Option A: Professional Health Worker, CHW-Mediated, Telemedicine Track}

4A. Conduct a validation pilot to assess the efficacy and potential of a sustainable telemedicine venture. This will include a validation strategy for obtaining intra-observer agreement rates for the client-CHW-healthcare professional network that will result in a better delivery of healthcare.

- Have a CHW conduct consultations with a significantly sized group of clients to generate the information to be transmitted to a remote nurse or doctor

- Have the nurse or doctor meet face-to-face with all clients after the CHW consultation for a routine health check-up and to record their assessment.

- Several days later, give the same nurse or doctor a copy of every client's CHWperformed consultation for review and response generation, removing any specific identifiers from the nurse's or doctor's original interaction with the client.

- Determine the intra-observer agreement rates by comparing the chief complaint, diagnosis, and feedback from the face-to-face assessment to the telemedicine assessment. If this is determined to be successful, move on to the next step; if not, refine the consultation and provide further training for the workers.

5A. Set specific payment plans for the healthcare professionals and establish the feasibility of maintaining sustainable telemedicine services.

6A. Conduct a rural pilot that validates the working business model for rural operations. Data gathered from the number consultations further contribute to the pilot, as do the number of individuals relayed to health clinics. An alternative would be an urban pilot similar to the rural pilot where operations are conducted in a larger town or city. Here, a local manager that coordinates a central location for $\mathrm{CHW}$ operations would be required as well.

7A. Start to charge per consultation once it is clear that the CHWs understand how to operate the venture and that the nurses or doctors are also running their side of the operation successfully.

8A. If operations are not conducive to economic sustainability, transition the business model to the Independent CHW Service Track. This could happen as a result of expensive professional healthcare workers or ineffective telecommunications. 
4B. Conduct rural or urban validation pilots. The purpose of this is to establish and validate a working business model for this track in select locations. Steps should include:

- Implement the specific business model developed with stakeholders. During this trial, clients are not charged for services and CHWs are paid a small stipend to incentivize them to provide services.

- Gather data on effectiveness for customers and CHWs. This includes measuring how many consultations CHWs have in a day and in a week, and how many of those consultations result in a client seeking medical attention from a health professional.

- If business model is successful, figure out if and how you can start charging customers for services. This fee must consider how much target clients can afford to spend on health care services and how much of a fee is needed to provide CHWs a sufficient income.

- Reduce CHW stipend and transition into a new payment plan. This payment plan could be to pay CHWs per consultation, per day of work, or transportation costs. The main goal of this step is to provide incentive for the CHWs to expand the venture's services and notoriety while progressing towards a subsidy-free business model

- Establish a local manager and phase out your organization's presence. This phase should be done slowly and with much monitoring to ensure that the venture can be independently sustainable without subsidies and oversight. Train this manager to ensure transparency, accountability, and open communication channels between them and everyone involved in the community health venture.

- Make sure profits are being reinvested into the venture itself and not left to the discretion of the local managers.

- Employees have to be properly incentivized in the pilot stage and beyond to avoid a high turnover rate because there is a high demand and low supply of health professionals in the developing world. The incentive should be adequate to maintain dedicated individuals but avoid attracting those with purely financial motivations. As both employees and the community as a whole understands the venture and work expectations improve, turnover rate should lessen.

\section{PART 4 OF 5: PREPARE FOR VENTURE LAUNCH}

Between piloting and launching the venture, there are several items to consider to improve the chances for venture success. The steps below review six tasks to consider during this time period.

1. Re-evaluate business model and operations. During this step, any problems that impact the venture's success must be addressed. It is advised to pursue changes in the business model that improve efficiency, accuracy, or profitability. 
2. Obtain all official permissions and agreements with local stakeholders and the health ministry for collaboration, such as written documents or emails stating that the business can operate in the country and documentation of contributions from partner groups.

3. Certify a permanent operation site. This site should be accessible to most of the clientele and to health professionals/CHWs. It should also provide the necessary resources and room required to perform consultations. This may come in the form of a permit for selling the services in the streets. Draft documents and have owners of involved facilities and the local manager of the venture sign.

4. Continue advertising and working with the community. Marketing and community engagement ensure the growth of the venture and also that appropriate services are being offered to the community. This should include:

- Discussion with leaders about more effective advertising methods.

- Walking through the village and meeting community members to develop community interest and trust. By establishing a connection between customers and the surrounding communities, community health systems can better market themselves as both a health service and as a community resource that people can leverage for a variety of different needs.

- Marketing schemes, such as subsidizing the first service, incentives for referrals, or discounts on certain days may also help generate the initial connection between the community health system and the customer.

- Since community health systems sometimes use text messaging as a method for communication, consistent messaging that is well-aligned with the brand helps grow the customer base while building trust with them.

- Formalizing a partnership with a strategic partner, such as a telecommunications company to initiate broader marketing

5. Consider community-based approaches to overcome social stigmas that may be preventing people from seeking health.

\section{Address potential legal concerns.}

- Seek legal counsel to determine if creating an official, legal entity in the country of operation fulfils the purposes of the venture. It may seem that forming a legal entity is cumbersome for a pilot phase, but it is important to weigh the benefits and drawbacks of creating a legal entity. Issues such as employee protection, mandated holidays, and taxation exist to ensure the social benefit of community members.

- Properly communicate the legal obligations of employees and those implementing the system to ensure that everyone has a thorough understanding of their responsibilities.

- Clearly separate the research and operational aspects of the system. In many countries, official research requires national approval, which can cost thousands of dollars. If research is taking place, whether it is tangential to the system or directly 
measuring its impact, it needs to be clearly divided from business operations. This distinction needs to be clearly explained during meetings with government officials.

\section{- Ensure that the health data is stored in a safe and legal manner.}

\section{PART 5 OF 5: EXECUTE VENTURE}

As you launch into the execution stage of your venture, evaluation, improvements, maintenance, and leadership are crucial. These steps explain how to take care of considerations during initial venture execution.

1. Evaluate success of venture on regular three-month intervals. This assessment could include measuring and ensuring:

- Increasing number of consultations

- Income generation for CHWs and nurses

- Clients successfully referred to hospitals and clinics

- Continued partnerships

- Positive changes in regional health status: this will be measured in different ways, depending on the targeted health problem. For example, if non-communicable diseases like high blood pressure are being targeted, self-reported dietary changes can be tracked.

2. Replace broken health care devices if necessary. Identify local manufacturers from whom health care devices could be bought, or find an inexpensive manufacturer from which to import devices.

3. Find local stakeholder to manage the purchasing and maintenance of appropriate devices. This should occur after the venture successfully develops and as the venture is being made independently sustainable. This step involves identifying local mechanics that can provide maintenance for health care devices.

4. Hire manager to oversee operations in region. Distinct from the local manager, this regional manager will provide oversight for all of the CHW operations and will ensure that devices can be more readily replaced. This manager will also allow from better and quicker responses to worker feedback on the venture.

\section{CONCLUSION}

With the rapid rise in the number of community health ventures to combat the large health inequities throughout the developing world, it is of the utmost importance that such programs are implemented properly in collaboration with the local community and government. ${ }^{11}$ Based upon 10 years of experience running a community health venture in Kenya with many challenges along the way, the authors have developed this framework for implementing a venture that can prove sustainable and effective. As opposed to a traditional hypothesis-driven research article or an application case-study, this article is a "how-to" in response to requests received from other 
International Journal for Service Learning in Engineering,

Humanitarian Engineering and Social Entrepreneurship

Vol. 11, No. 2, pp. 72-86, Fall 2016

ISSN 1555-9033

innovators and communities on setting up telemedicine systems. While it is not possible to capture every aspect of the process of validating and operationalizing such systems, this article hopes to help innovators get started and learn about some of the unknowns that need to be considered.

The framework presented in this article should be used as a set of guidelines for setting up a community health program and is adaptable to fit specific contexts and populations. Many community health ventures today face the challenge of advancing beyond the pilot stage and scaling after the first several years. With future directions for a venture integral to the framework developed in this article, utilization of this framework should assist in minimizing failures. Finding newer and better ways of providing increased access to health care for individuals in developing countries is paramount, and leveraging existing resources (such as $\mathrm{CHWs}$ ) is certainly among the most promising solutions.

\section{ACKNOWLEDGMENT}

The authors would like to thank Rachael Dzombak and Jeff Lackey for their wisdom, input, and years of field experience designing, developing, and implementing Mashavu through their involvement in HESE.

\section{REFERENCES}

1. Global Health Workforce Alliance. 2010. "Global Experience of Community Health Workers for Delivery of Health Related Millennium Development Goals: A Systematic Review, Country Case Studies, and Recommendations for Integration into National Health Systems". Report, World Health Organization.

2. Singh, P. and Chokshi, D. 2013. "Community Health Workers - A Local Solution to a Global Problem.” The New England Journal of Medicine 369: 894-896.

3. Center for Health Market Innovations. 2016. "Djantoli." http://healthmarketinnovations.org/program/djantoli (Accessed 12 January 2016).

4. Chen, S., Cheng, A., \& Mehta, K. 2013. A review of telemedicine business models. Telemedicine and e-Health, 19(4), 287-297.

5. Wootton, R. 2008. "Telemedicine support for the developing world." Journal of Telemedicine and Telecare 14 (3): 109-114.

6. Lackey, J. D., Suffian, S., Dzombak, R., \& Mehta, K. 2015. Demonstrating demand for preventive health services in rural Kenya. Journal of Humanitarian Engineering, 3(1), 8-16.

7. Kodukula, S., and Nazvia, M. 2011. "Evaluation of Critical Success Factors for Telemedicine Implementation." International Journal of Computer Applications 12 (10): 29-36.

8. Fleishman, A., Wittig, J., Milnes, J., Baxter, A., Moreau, J., \& Mehta, K. 2010. Validation Process for a Social Entrepreneurial Telemedicine Venture in East Africa. International Journal for Service Learning in Engineering, 5(1), 1-24.

9. Holmes, K., Suffian, S., Lackey, J., \& Mehta, K. 2014. Pilot results of a telemedicine social franchise in rural Kenya. Humanitarian Technology: Science, Systems and Global Impact 2014. Elsevier Ltd.

10. Callan, J., Sundin, P., Suffian, S., \& Mehta, K. 2014. Designing sustainable revenue models for CHW-centric entrepreneurial ventures. Global Humanitarian Technology Conference (pp. 687693). IEEE.

11. Gorski, I.; Bram, J.; Sutermaster, S.; Eckman, M.; Mehta, K. (Aug 2016). Typology of mHealth Projects' Value Propositions. Journal of Medical Engineering \& Technology. 